\title{
TRANSLATES AND MULTIPLIERS OF ABELIAN DIFFERENCE SETS
}

\author{
ROBERT L. MCFARLAND AND BART F. RICE
}

\begin{abstract}
It is shown that every abelian difference set has a translate which is fixed by all numerical multipliers. If an abelian difference set in a group of order $v$ has numerical multipliers $t_{1}, \ldots, t_{m}$ which satisfy $\operatorname{gcd}\left(t_{1}-\right.$ $\left.1, \ldots, t_{m}-1, v\right)=1$, then there is a unique translate which is fixed by all multipliers.
\end{abstract}

1. Introduction. Let $G$ be a multiplicative group of finite order $v$. A subset $D:=\left\{d_{1}, \ldots, d_{k}\right\}$ of $G$ is a difference set in $G$ with parameters $(v, k, \lambda)$ in case the aggregate of "differences", $d_{i}^{-1} d_{j}(i, j=1, \ldots, k, i \neq j)$, replicates each nonidentity element of $G$ exactly $\lambda$ times. We assume $0<\lambda<k<v-$ 1 to avoid the trivial difference sets which have $k=0,1, v-1$ or $v$. The difference set is cyclic, abelian or nonabelian if the group $G$ is cyclic, abelian or nonabelian, respectively. For additional information on difference sets see Baumert [1], Hall [3, Chapter 11] or Mann [4, Chapters 6, 7, 8].

For any $g$ in $G$ we call $D g:=\left\{d_{1} g, \ldots, d_{k} g\right\}$ a translate of $D$. Note that $D g$ is also a difference set. Furthermore, $D g=D$ if and only if $g=1$, for otherwise there would be $k(>\lambda)$ replications of $g$ among the "differences" $d_{i}^{-1} d_{j}$.

If $\sigma: g \mapsto g^{\sigma}$ is an automorphism of $G$, define $D(\sigma):=\left\{d_{1}^{\sigma}, \ldots, d_{k}^{\sigma}\right\}$. If $D(\sigma)$ is a translate of $D$, then $\sigma$ is called a multiplier of $D$. The multiplier $\sigma$ fixes the translate $D g$ in case $(D g)(\sigma)=D g$. If there is an integer $t$ such that the action of the multiplier $\sigma$ on $G$ is $\sigma: g \mapsto g^{t}$, then we identify $t$ and $\sigma$ and call $t$ a numerical multiplier; otherwise $\sigma$ is a nonnumerical multiplier. The multipliers of $D$ constitute a subgroup of the automorphism group of $G$, called the multiplier group of $D$. The numerical multipliers are a subgroup of the center of the multiplier group.

In this paper we consider the question: Must an abelian difference set have a translate which is fixed by all of its multipliers? The answer is known to be yes if the parameters $v$ and $k$ of the difference set are relatively prime or if there is a numerical multiplier $t$ such that $t-1$ is prime to $v$. A difference set in an elementary abelian group of order 16 shows that the answer is not always yes.

We prove that such a fixed translate exists for all numerical multipliers.

Received by the editors May 30, 1975 and, in revised form, April 7, 1977.

AMS (MOS) subject classifications (1970). Primary 05B 10.

Key words and phrases. Difference set, multiplier. 
Thus, in particular, a cyclic difference set always has a translate which is fixed by all multipliers. We also give a new condition for an abelian difference set to have a translate which is fixed by all multipliers.

2. Fixed translates. It is well known, Mann and McFarland [5], that a multiplier of a difference set must fix at least one translate. Indeed, a multiplier of a difference set $D$ in a group $G$ induces the same orbit structure on the translates of $D$ as it induces on the elements of $G$, cf. Parker [6]. The number of translates fixed by a multiplier $\sigma$ is the order of the subgroup $\left\{g \in G: g^{\sigma}=g\right\}$.

J. Jans (see Hall [3, p. 140]) has noted that if the parameters of an abelian difference set $D$ satisfy $\operatorname{gcd}(v, k)=1$, then there is a unique translate $D g$ for which the product of the elements of $D g$ is 1 , and this translate is fixed by all multipliers. In this situation there can be other translates which are also fixed by all multipliers. For example, the cyclic difference set with parameters $(v, k, \lambda)=(57,8,1)$ has exactly three translates which are fixed by the multiplier 7 which is a generator of the multiplier group.

The case $m=1$ of the following theorem is proved in [5] and in [4, p. 81].

THEOREM 1. Suppose a difference set $D$ in an abelian group $G$ of order $v$ has (some) numerical multipliers $t_{1}, \ldots, t_{m}$ which satisfy

$$
\operatorname{gcd}\left(t_{1}-1, \ldots, t_{m}-1, v\right)=1 .
$$

Then $D$ has a unique translate which is fixed by every multiplier.

Proof. Let $D\left(t_{i}\right)=D g_{i}(i=1, \ldots, m)$. Then $D\left(t_{i} t_{j}\right)=D\left(t_{j} t_{i}\right)$ implies that

$$
g_{i}^{t_{j}-1}=g_{j}^{t_{i}-1} \quad(i, j=1, \ldots, m) .
$$

There are integers $a_{1}, \ldots, a_{m}$ such that

$$
a_{1}\left(t_{1}-1\right)+\cdots+a_{m}\left(t_{m}-1\right) \equiv 1(\bmod v) .
$$

Let $g=g_{1}^{a_{1}} \cdots g_{m}^{a_{m}}$. Then

$$
g^{t_{j}-1}=\prod_{i=1}^{m} g_{i}^{a_{i}\left(t_{j}-1\right)}=\prod_{i=1}^{m} g_{j}^{a_{i}\left(t_{i}-1\right)}=g_{j} .
$$

Therefore, the translate $D_{1}:=D g^{-1}$ is fixed by each of $t_{1}, \ldots, t_{m}$. Suppose $D_{1} h$ is also fixed by $t_{1}, \ldots, t_{m}$. Then $\left(D_{1} h\right)\left(t_{i}\right)=D_{1} h$ implies that $h^{t_{i}-1}=1$ $(i=1, \ldots, m)$. Thus

$$
h=h^{a_{1}\left(t_{1}-1\right)+\cdots+a_{m}\left(t_{m}-1\right)}=1 .
$$

Hence $D_{1}$ is the only translate which is fixed by each of $t_{1}, \ldots, t_{m}$. Let $\sigma$ be any multiplier and suppose $D_{1}(\sigma)=D_{1} h$. Then $D_{1}\left(\sigma t_{i}\right)=D\left(t_{i} \sigma\right)$ implies that $h^{t_{i}-1}=1$. Hence $h=1$ as above, so $D_{1}$ is fixed by every multiplier.

Dembowski $[2$, p. 85$]$ notes that in general an abelian difference set need not have a translate which is fixed by all its multipliers. For example, let $G$ be an elementary abelian group of order 16 with generators $a, b, c, d$. Then $D=\{1, a, b, c, d, a b c d\}$ is a difference set in $G$ with parameters $(v, k, \lambda)=$ $(16,6,2)$. The automorphism $\alpha$ defined by $\alpha: a \mapsto b \mapsto c \mapsto d \mapsto a$ is a 
multiplier which fixes $D$. The automorphism $\beta$ defined by $\beta$ : $(a \mapsto a b c d$, $b \mapsto b c d, c \mapsto a c d, d \mapsto a b d)$ is a multiplier since $D(\beta)=D a b c d$. The automorphism group generated by $\alpha$ and $\beta$ is transitive on the nonidentity elements of $G$, so no translate of $D$ can be fixed by both $\alpha$ and $\beta$.

To prove the next theorem we introduce the group ring $\mathbf{Z} G$ of the finite multiplicative abelian group $G$ over the ring $\mathbf{Z}$ of integers. We identify any subset $S$ of $G$ with its characteristic function in $\mathbf{Z} G$, i.e., $S=\sum_{g \in G} a_{g} g$, where the coefficients $a_{g}=1$ or 0 according to whether $g \in S$ or $g \notin S$. In particular, a difference set $D$ in $G$, an element $g \in G$ or $G$ itself can be considered elements of $\mathbf{Z} G$. However, we write $G(1)$ to denote the characteristic function of $G$. For any element $A \in \mathbf{Z} G$,

$$
A:=\sum_{g \in G} a_{g} g, \quad a_{g} \in \mathbf{Z},
$$

and any integer $t$ we define

$$
A(t):=\sum_{g \in G} a_{g} g^{t}
$$

Thus in the group ring notation, an integer $t$ is a multiplier of the difference set $D$ in case $D(t)=D g$ for some group element $g$. The difference set property implies that

$$
D(-1) D=(k-\lambda) 1_{G}+\lambda G(1),
$$

where $k, \lambda$ are those parameters of $D$ and $1_{G}$ is the identity of $G$.

We also utilize the concept of characters of $G$ acting on $\mathbf{Z} G$ and the inversion formula which expresses the coefficients of an element $A \in \mathbf{Z} G$ in terms of the values the characters take on $A$. In particular, if $A, B$ are two elements of $\mathbf{Z} G$ such that $\chi(A)=\chi(B)$ for all characters $\chi$, then $A=B$. For details see Mann [4, pp. 73-75].

THEOREM 2. Every abelian difference set has a translate which is fixed by all of its numerical multipliers.

Proof. Let $D$ be a difference set in the finite abelian group $G$. Express $G$ as the direct product $G=G_{1} \times \cdots \times G_{m}$, where each $G_{i}$ is a cyclic group of prime power order. Let $D_{i}$ be the image of $D$ under the natural group ring epimorphism $\mathbf{Z} G \rightarrow \mathbf{Z} G_{i}$ induced by the canonical group epimorphism $\alpha_{i}$ : $G \rightarrow G_{i}$. We assert that each $G_{i}$ contains an element $g_{i}$ such that the translate $D_{i} g_{i}$ is fixed by every numerical multiplier of $D$. Let $T$ be the group of numerical multipliers of $D$ and let $T_{i}$ be the multiplier group for $D_{i}$ obtained by reducing the elements of $T$ modulo the order of $G_{i}$. First suppose $T_{i}$ is cyclic. Let $t \in T$ be a generator of $T_{i}$ and let $D g$ be a translate fixed by $t$. Let $\alpha_{i}: g \mapsto g_{i}$. Then $D_{i} g_{i}$ is fixed by $t$. Hence $D_{i} g_{i}$ is fixed by every element of $T_{i}$ and thus also fixed by every element of $T$.

Now suppose $T_{i}$ is not cyclic. The automorphism group $\mathbb{Q}_{i}$ of $G_{i}$ is noncyclic only if the order of $G_{i}$ is $2^{e}$ for some $e>3$, and then $\mathscr{Q}_{i}$ is isomorphic to the direct product of a group of order 2 with a cyclic group of 
order $2^{e-2}$. Any noncyclic subgroup of $\mathbb{Q}_{i}$ must contain all three elements of $Q_{i}$ which have order 2 . In particular, $T_{i}$ contains -1 . Thus $T$ contains an element $t \equiv-1\left(\bmod 2^{e}\right)$. Let $D g$ be a translate fixed by this $t$. Let $\alpha_{i}: g \mapsto g_{i}$ and let $E_{i}:=D_{i} g_{i}$. Then $E_{i}(-1)=E_{i}$. Hence

$$
E_{i}^{2}=E_{i}(-1) E_{i}=(k-\lambda) 1_{i}+\lambda\left[G: G_{i}\right] G_{i}(1),
$$

where $k, \lambda$ are those parameters of $D, 1_{i}$ is the identity of $G_{i}$ and [ $G: G_{i}$ ] is the index of $G_{i}$ in $G$. Each character $\chi$ of $G_{i}$ can be extended linearly to yield a homomorphism from $\mathbf{Z} G_{i}$ into the order $\mathbf{Z}[\zeta]$ generated by a primitive $2^{e}$ th root of unity $\zeta$. If $\chi_{0}$ is the principal character, then $\chi_{0}\left(E_{i}\right)=k$. If $\chi$ is a nonprincipal character of $G_{i}$, then $\chi\left(G_{i}(1)\right)=0$, so

$$
\chi\left(E_{i}\right)^{2}=\chi\left(E_{i}^{2}\right)=k-\lambda .
$$

Since the order of $G$ is even, the well-known Bruck-Ryser-Chowla conditions imply that $k-\lambda$ is a square. Hence $\chi\left(E_{i}\right) \in \mathbf{Z}$ for every character $\chi$. For every odd integer $s$ let $\sigma_{s}$ be the automorphism of $\mathbf{Z}[\zeta]$ defined by $\sigma_{s}: \zeta \mapsto \zeta^{s}$. Then

$$
\chi\left(E_{i}(s)\right)=\sigma_{s} \chi\left(E_{i}\right)=\chi\left(E_{i}\right)
$$

for every character $\chi$ of $G_{i}$. Hence by the inversion formula for $\mathbf{Z} G_{i}$, $E_{i}(s)=E_{i}$. That is, every odd integer $s$ is a multiplier which fixes $E_{i}$. Since the order of $G$ is even, $T$ contains no even integers. Thus $E_{i}=D_{i} g_{i}$ is fixed by every element of $T$.

Use the values of $\dot{g}_{i}$ specified above to define $g:=\left(g_{1}, \ldots, g_{m}\right) \in G_{1}$ $\times \cdots \times G_{m}$. Let $t \in T$ and suppose $(D g)(t)=D h$, where $h$ $=:\left(h_{1}, \ldots, h_{m}\right)$. Apply the group ring epimorphism induced by $\alpha_{i}$ to obtain $\left(D_{i} g_{i}\right)(t)=D_{i} h_{i}$. But $t$ fixes $D_{i} g_{i}$, so $D_{i} g_{i}=D_{i} h_{i}$. Multiply this equation by $D_{i}(-1)$ to see that $g_{i}=h_{i}$. Thus $g=h$ so the translate $D g$ is fixed by every numerical multiplier.

Note that the proof of Theorem 2 is not valid for nonnumerical multipliers, since nonnumerical multipliers will not necessarily commute with the epimorphisms $\alpha_{i}$.

Since a cyclic group has only "numerical automorphisms", we have

COROLlARY. Every cyclic difference set has a translate which is fixed by all of its multipliers.

The authors wish to thank John F. Dillon for pointing out to them their common interest in the problems considered in this paper.

\section{REFERENCES}

1. L. D. Baumert, Cyclic difference sets, Lecture Notes in Math., vol. 182, Springer-Verlag, New York, 1971.

2. P. Dembowski, Finite geometries, Springer-Verlag, New York, 1968.

3. M. Hall, Jr., Combinatorial theory, Blaisdell, Waltham, Massachusetts, 1967.

4. H. B. Mann, Addition theorems, Wiley, New York, 1965. 
5. H. B. Mann and R. L. McFarland, On multipliers of difference sets, Canad. J. Math. 17 (1965), 541-542.

6. E. T. Parker, On collineations of symmetric designs, Proc. Amer. Math. Soc. 8 (1957), 305-351.

Department of Mathematics, Wright State University, Dayton, Oho 45435

Department of Defense, Fort Grorge G. Meade, Markland 20755 